BULLETIN OF THE

AMERICAN MATHEMATICAL SOCIETY

Volume 78, Number 1, January 1972

\title{
RELATIVE IMAGINARY QUADRATIC FIELDS OF LOW CLASS NUMBER
}

\author{
BY LARRY JOEL GOLDSTEIN ${ }^{1}$ \\ Communicated by Oscar Goldman, June 22, 1971
}

\begin{abstract}
Let $K$ be a normal totally real algebraic number field. Then it is possible to effectively determine all totally imaginary quadratic extensions of $K$ of class number 1 . A partial result for class number 2 is obtained.
\end{abstract}

Let $K$ be a totally real algebraic number field and let $h$ be a positive integer. It is a simple consequence of the Brauer-Siegel theorem [2] that there exist only finitely many totally imaginary quadratic extensions $L$ of $K$ having class number $h$. In this note, we will announce some results connected with the following:

Problem. For fixed $K$, determine effectively all totally imaginary quadratic extensions of class number $h$.

When $K=\boldsymbol{Q}$, this problem is equivalent to the determination of an effective procedure for classifying all imaginary quadratic fields of class number $h$. Even in this special situation, little is known. In 1966, Stark [4] settled the case $h=1$ by proving that there are precisely 9 imaginary quadratic fields of class number 1. In 1970, the author [3], Baker [1], and Stark [5] succeeded in giving an effective upper bound for the absolute value of the discriminant of an imaginary quadratic field of class number 2 . At the present time, the solution to the problem, for $K=Q, h \geqq 3$, is not known.

Recently, J. Sunley [6] has proved the following result:

THEOREM [S]. Let $K$ be a fixed totally real algebraic number field and let $L$ be a totally imaginary quadratic extension of $K$, having class number $h_{L}$ and discriminant $d_{L}$. There exists an effectively computable constant $c=c(K, h)$ such that if $h=h_{L}$, then

$$
\left|d_{L}\right| \leqq c,
$$

with the possible exception of one field $L$.

Our main results concern the possible exceptional field $L$.

THEOREM 1. Let all notations be as in [S], and assume that $K$ is normal. Then the exceptional field $L$ (if it exists) must be normal over $\boldsymbol{Q}$.

AMS 1969 subject classifications. Primary 1065; Secondary 1068.

Key words and phrases. Number field, class number, imaginary quadratic extension.

${ }^{1}$ Research supported by NSF Grant GP-20538. 
THEOREM 2. Let all notations be as in [S], and assume that $K$ and $L$ are normal.

(1) If $h_{L}=1$, then either $L=K\left((-p)^{1 / 2}\right)$, where $Q\left((-p)^{1 / 2}\right)$ is an imaginary quadratic field of class number 1 , or L belongs to a finite, effectively determined collection of fields.

(2) Let $K$ be a real quadratic field of class number 1 and fundamental unit of norm $=-1$. If $h_{L}=2$, then either $L=K\left((-p q)^{1 / 2}\right)$, where $Q\left((-p q)^{1 / 2}\right)$ is an imaginary quadratic field of class number 2 , or $L$ belongs to a finite, effectively determined collection of fields.

By combining Theorems [S], 1 and 2, with the solutions to the problem for $K=Q, h=1,2$, we get the following results.

THEOREM 3. Assume that $K$ is normal. It is possible to effectively determine all totally imaginary quadratic extensions $L$ of $K$ having class number 1.

THEOREM 4. Let $K$ be a real quadratic field of class number 1 whose fundamental unit has norm $=-1$. Then it is possible to effectively determine all totally imaginary quadratic extensions $L$ of $K$ having class number 2.

\section{BIBLIOGRAPHY}

1. A. Baker, Imaginary quadratic fields of class number 2, Ann. of Math. (to appear).

2. R. Brauer, On the zeta-functions of algebraic number fields, Amer. J. Math. 69 (1947), 243-250. MR 8, 567.

3. L. Goldstein, Imaginary quadratic fields of class number 2, J. Number Theory (to appear).

4. H. M. Stark, A complete determination of the complex quadratic fields of class-number one, Michigan Math. J. 14 (1967), 1-27. MR 36 \# 5102.

5. - Imaginary quadratic fields of class number 2, Ann. of Math. (to appear).

6. J. Sunley, On the class numbers of totally imaginary quadratic extensions of totally real fields, Thesis, University of Maryland, College Park, Md., 1971.

Department of Mathematics, University of Maryland, College Park, Maryland 20742 